\title{
Peroxiredoxin 4, a new oxidative stress marker in follicular fluid, may predict in vitro fertilization and embryo transfer outcomes
}

\author{
Qian Yi ${ }^{1}$, Chao Meng ${ }^{2}$, Ling-Bo Cai ${ }^{1}$, Yu-Gui Cui ${ }^{1}$, Jia-Yin Liu $^{1}$, Yan Meng ${ }^{1}$ \\ ${ }^{1}$ The State Key Laboratory of Reproductive Medicine, Clinical Center of Reproductive Medicine, The First Affiliated Hospital, Nanjing Medical \\ University, Nanjing, China; ${ }^{2}$ Department of Critical Care Medicine, Nanjing First Hospital, Nanjing Medical University, Nanjing, China \\ Contributions: (I) Conception and design: Y Qian, Y Meng; (II) Administrative support: YG Cui, JY Liu; (III) Provision of study materials or patients: \\ Q Yi, C Meng, Y Meng; (IV) Collection and assembly of data: Q Yi, LB Cai; (V) Data analysis and interpretation: Q Yi, C Meng, Y Meng; (VI) \\ Manuscript writing: All authors; (VII) Final approval of manuscript: All authors. \\ Correspondence to: Yan Meng. The State Key Laboratory of Reproductive Medicine, Clinical Center of Reproductive Medicine, The First Affiliated \\ Hospital, Nanjing Medical University, Nanjing, China. Email: ctmengyan@hotmail.com.
}

Background: Peroxiredoxin $4(\operatorname{Prdx} 4)$, a member of the Prdx family, can catalyze the reduction of reactive oxygen species. This study aims to explore whether $\operatorname{Prdx} 4$ can serve as an effective marker in follicular fluid (FF) for predicting in vitro fertilization/intracytoplasmic sperm injection (IVF/ICSI) cycle outcomes.

Methods: In this prospective study, all participants were recruited from the center of clinical reproductive medicine from 2017 September to 2018 December. Women with tubal or male factor infertility undergoing their first IVF/ICSI cycle were recruited $(n=138)$. FF samples from each patient were collected on the day of oocyte retrieval. $\operatorname{Prdx} 4$ concentrations were measured, and the correlation between $\operatorname{Prdx} 4$ levels and IVF outcomes was analyzed.

Results: The results showed that pregnant women had higher levels of $\operatorname{Prdx} 4$ than nonpregnant women. $\operatorname{Prdx} 4$ was positively correlated with the oocyte fertilization rate $(\mathrm{r}=0.334 ; \mathrm{P}=0.011)$ and good quality embryo rate $(\mathrm{r}=0.326 ; \mathrm{P}=0.013)$. Furthermore, we found that the clinical pregnancy rate was positively correlated with Prdx4 levels in a concentration-dependent manner in the Prdx4 quartiles $(<13.38,13.83-$ $16.93,16.93-22.93,>22.93 \mathrm{ng} / \mathrm{mL})$. The fertilization rates, clinical pregnancy rates and live pregnancy rates were all significantly higher in the highest $\operatorname{Prdx} 4$ quartile group than in the lowest quartile. Moreover, the results indicated that $\operatorname{Prdx} 4$ had an area under the receiver operating characteristic curve (AUC) of 0.754 , corresponding to an optimal cutoff point of $22.30 \mathrm{ng} / \mathrm{mL}$.

Conclusions: Our results provide evidence that higher expression of antioxidants, such as $\operatorname{Prdx} 4$, in the FF of IVF patients tends to indicate a higher likelihood of pregnancy through an oocyte quality mechanism.

Keywords: Follicular fluid (FF); oocyte quality; ovarian stimulation; oxidative stress; peroxiredoxin $4(\operatorname{Prdx} 4)$

Submitted Jan 14, 2020. Accepted for publication Jul 21, 2020.

doi: $10.21037 /$ atm-20-397

View this article at: http://dx.doi.org/10.21037/atm-20-397

\section{Introduction}

Approximately $9 \%$ of women of reproductive age worldwide suffer from infertility, and in vitro fertilization-embryo transfer (IVF-ET) technology offers the highest success rate for infertile patients (1). However, nearly $75 \%$ of IVF cycles do not result in a successful pregnancy, and more cycles are needed after IVF failure, which negatively impacts couples. Therefore, effective biomarkers for predicting the probability of pregnancy with IVF treatment are urgently needed. A positive outcome of an IVF procedure requires the successful occurrence of several events, such as oocyte maturation, ovulation and fertilization, especially oocyte quality, which plays a key role in the whole process. Follicular fluid (FF) is a major component of the biological environment that supports oocyte development and subsequent embryo development during IVF $(2,3)$. 
The components of FF include various mediators, such as cytokines, growth factors, regulatory molecules, reactive oxygen species (ROS) and antioxidants (4). These mediators have direct effects on the quality and maturation ability of oocytes.

Peroxiredoxin $4(\operatorname{Prdx} 4)$ is a member of the peroxiredoxin antioxidant enzyme family, whose members catalyze the reduction of ROS, such as $\mathrm{H}_{2} \mathrm{O}_{2}$ and various organic hydroperoxides, to form water and alcohols (5). In our previous studies, cellular $\operatorname{Prdx} 4$ was found to be expressed in the mouse cumulus-oocyte complex (COC) and human ovary (6). Moreover, we demonstrated that $\operatorname{Prdx} 4$ was expressed at lower levels in polycystic ovaries than in normal ovaries and that the expression of $\operatorname{Prdx} 4$ in the granulosa cells (GCs) of mature follicles was higher than that in the GCs of immature follicles (6). These observations suggested that cellular $\operatorname{Prdx} 4$ may play a role in follicular development. Many studies have identified $\operatorname{Prdx} 4$ as an antioxidant and suggested that the $\operatorname{Prdx} 4$ expression level might be an effective biomarker of oxidative stress (OS) in the body and that $\operatorname{Prdx} 4$ expression can predict the incidence of several diseases, such as cardiovascular disease and recurrent pregnancy loss (7-9). However, the role of $\operatorname{Prdx} 4$ in the female reproductive system, especially in females undergoing IVF, is still unknown.

The aim of this study was to investigate the relationship between $\operatorname{Prdx} 4$ levels in the FF of infertility patients and subsequent oocyte quality and IVF-ET outcomes, especially the clinical pregnancy rate. These results may provide clinically relevant information for predicting IVF outcomes and reveal the mechanism of $\operatorname{Prdx} 4$ activity in oocyte development. We present the following article in accordance with the STROBE reporting checklist (available at http://dx.doi.org/10.21037/atm-20-397).

\section{Methods}

This prospective clinical cohort study was conducted between 2017 September and 2018 December at the reproductive clinical center of The First Affiliated Hospital of Nanjing Medical University. The study was conducted in accordance with the Declaration of Helsinki (as revised in 2013). This study was approved by the Institutional Ethics Committee of The First Affiliated Hospital of Nanjing Medical University (No. 2016-SRFA-038), and informed consent was obtained from all patients. A total of 138 infertility patients diagnosed with tubal or male factor infertility were recruited for this study. The exclusion criteria were as follows: $\geq 32$ years of age; body mass index (BMI) $\geq 24 \mathrm{~kg} / \mathrm{m}^{2}$; primary ovarian insufficiency; polycystic ovary syndrome; cycles with the dominant FF contaminated with blood during oocyte retrieval; cycles with the dominant FF not yielding oocytes; or any history of other gynecological or other endocrine diseases (thyroid disease, diabetes mellitus, or Cushing's syndrome). The FF samples were obtained from patients who first underwent either IVF or intracytoplasmic sperm injection (ICSI).

\section{COH, IVF-ET and follicular fluid collection}

All participants were injected with the gonadotropinreleasing hormone (GnRH) agonist Decapeptyl/Triptorelin (Ferring Pharmaceuticals Co., Ltd, Germany) starting from the mid-luteal phase. Once pituitary downregulation was achieved after 2 weeks, subjects were injected with recombinant follicle-stimulating hormone (FSH) (Gonal F, Serono, Geneva, Switzerland). The sizes of the follicles were monitored periodically via ultrasound and serum estradiol assays. When there were three or more follicles with a mean diameter $\geq 17 \mathrm{~mm}$, human chorionic gonadotropin (hCG) (Pregnyl, Organon, The Netherlands) was administered subcutaneously. Follicle size was estimated by ultrasound $36 \mathrm{~h}$ after the administration of hCG, and oocytes were aspirated. FF was carefully aspirated from follicles of optimal size (diameters in the range of $16-20 \mathrm{~mm}$ ) in each patient during oocyte retrieval, and the FF of each follicle from the same patient was combined. The FF was aspirated without contamination with the flushing medium or culture medium. Oocytes were isolated, and FF samples from the same patient were pooled and centrifuged at 2,000 rpm for $10 \mathrm{~min}$. Supernatants were stored at $-70{ }^{\circ} \mathrm{C}$ until analysis. FF contaminated with culture medium or blood was discarded.

\section{Assay of Prdx4 levels}

Prdx4 levels in FF were assessed using an enzyme-linked immunosorbent assay (ELISA) (Abnova, Taipei, Taiwan) according to the manufacturer's protocol as previously described (10).

\section{Hydrogen peroxide $\left(\mathrm{H}_{2} \mathrm{O}_{2}\right)$ assay and antioxidant activity}

$\mathrm{H}_{2} \mathrm{O}_{2}$ concentrations in $\mathrm{FF}$ were measured using a colorimetric hydrogen peroxide kit (Ann Arbor, MI, USA) as previously described. The levels of glutathione (GSH), superoxide dismutase (SOD), catalase (CAT) 


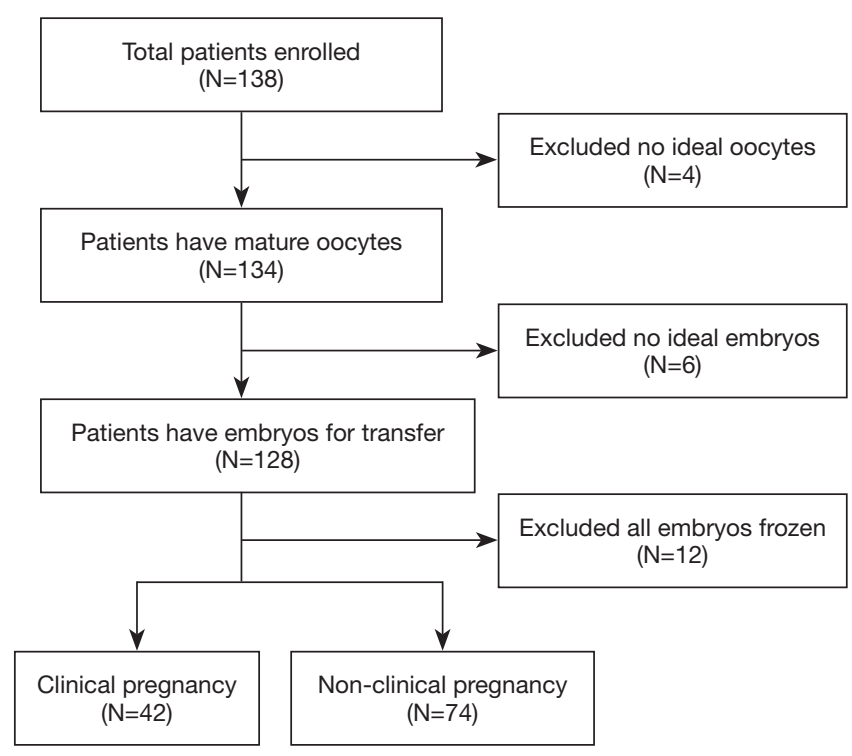

Figure 1 Flow chart of the study. The flow of analyses in this study and how exclusion criteria apply.

and malondialdehyde (MDA) activity were measured as previously described. Glutathione peroxidase (GSH-Px) activity was measured using an Amplex Red Peroxidase Assay Kit (Molecular Probes, USA). Briefly, $25 \mu \mathrm{L}$ of FF was dispensed in each well of a 96 well plate and mixed with $25 \mu \mathrm{L}$ of reaction buffer ( $0.05 \mathrm{M}$ sodium phosphate, $\mathrm{pH}$ 7.4). Then, $50 \mu \mathrm{L}$ of a working solution of Amplex Red reagent $(100 \mu \mathrm{m})$ and $\mathrm{H}_{2} \mathrm{O}_{2}(2 \mathrm{~mm})$ was added into each microplate well, and samples were incubated at room temperature for $30 \mathrm{~min}$ and protected from light. Absorbances were measured with a $412 \mathrm{~nm}$ filter (Spectra Shell Microplate Reader, SLT Spectra).

\section{Evaluation of parameters associated with oocyte quality}

Fertilization was evaluated $16-18 \mathrm{~h}$ after insemination, and embryo cleavage was evaluated 24 h later. Embryo morphology was assessed on day 3 of in vitro culture, with consideration of the number of cells and percentage of fragmentation. The variables related to oocyte quality were as follows: (I) the fertilization rate, namely, the proportion of 2 pronuclei $(\mathrm{PN})$ observed relative to the number of oocytes retrieved; (II) the cleavage rate, namely, the proportion of cleavage-stage embryos relative to the total number of $2 \mathrm{PN}$; and (III) the proportion of good quality embryos, namely, embryos containing at least 7 cells and with less than or equal to $10 \%$ fragmentation on day 3 postinsemination relative to the total number of $2 \mathrm{PN}$. One or two embryos were transferred 3 days after oocyte retrieval. The luteal phase was supported with $20 \mathrm{mg}$ of oral dydrogesterone (Duphaston, Abbott, USA) and $400 \mathrm{mg}$ of vaginal micronized progesterone (Utrogestan, Laboratoires Besins International, Paris, France) daily. Progesterone support was initiated on the day of oocyte retrieval and continued for 14 days; the treatment continued for another 8 weeks if a pregnancy was achieved. A clinical pregnancy was identified 4-5 weeks after oocyte retrieval by the presence of an intrauterine gestational sac and a pulsating fetal heartbeat. The live birth rate was defined as the number of deliveries that resulted in at least one live-born baby.

\section{Statistical analysis}

Data are presented as the mean \pm standard error of the mean (SEM) for experimental studies and the mean \pm standard deviation (SD) for demographic data. Data distribution was assessed for normality using the ShapiroWilk and Kolmogorov-Smirnov tests. Normally distributed data were analyzed with Student's $t$-test. The $\chi^{2}$ test was used for qualitative data. Univariate analysis was performed using a nonparametric Spearman test. A receiver operating characteristic (ROC) curve was drawn. The sensitivity and specificity of the test at the set cut-off values were calculated, and the area under curve (AUC) was estimated. $\mathrm{P}<0.05$ was considered statistically significant; $\mathrm{P}<0.01$ was considered highly significant.

\section{Results}

\section{Demographics and clinical data}

Twenty-two patients who did not undergo ET, ten of whom did not have oocytes or embryos of ideal quality, were excluded, while twelve patients had their embryos frozen due to ovarian hyperstimulation syndrome or other reasons (Figure 1). To further determine the relationship between OS marker levels in FF and IVF outcomes, we divided the patients into the clinically pregnant and nonclinically pregnant groups. The main demographics and clinical parameters of the patients are summarized in Table 1.

\section{Analysis of oxidative stress marker concentrations in pregnant versus nonpregnant patients}

We studied the relationship between OS marker levels 
Table 1 Demographic and biochemical data of patients according to clinical pregnancy outcome

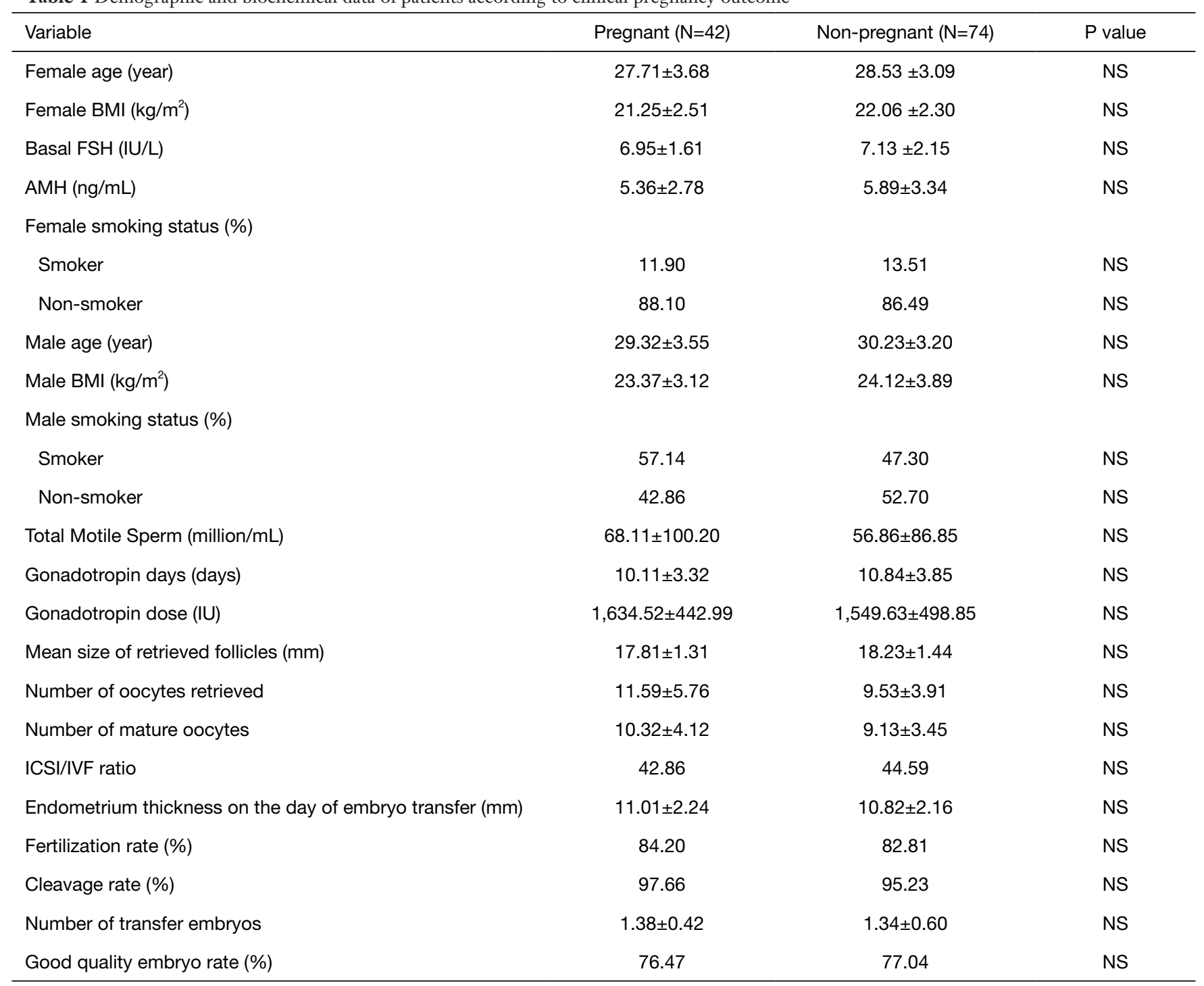

Values are mean $\pm S D$; ${ }^{*}$, statistically significant $(P<0.05)$. NS, not significant.

and IVF outcomes. There were no significant differences in the concentrations of ROS, such as $\mathrm{H}_{2} \mathrm{O}_{2}$, between the two groups. Additionally, no significant difference in the concentrations of GSH, a nonenzymatic antioxidant, was found between the two groups.

Interestingly, the levels of enzymatic antioxidants, such as GSH-Px, SOD and Prdx4, were all significantly higher in the clinically pregnant group (Table 2). The mean GSHPx level in the pregnant group was $130.95 \pm 10.56 \mathrm{U} / \mathrm{mL}$, while the mean GSH-Px level in the nonpregnant group was $100.83 \pm 5.40 \mathrm{U} / \mathrm{mL}(\mathrm{P}=0.012)$. The mean SOD level in $\mathrm{FF}$ was $54.06 \pm 3.61 \mathrm{U} / \mathrm{mL}$ in the pregnant group and
$43.87 \pm 1.76 \mathrm{U} / \mathrm{mL}$ in the nonpregnant group $(\mathrm{P}=0.011)$. Furthermore, the $\operatorname{Prdx} 4$ level was significantly higher in the pregnant group than in the nonpregnant group $(22.26 \pm 1.65$ vs. $16.43 \pm 0.97 \mathrm{ng} / \mathrm{mL}, \mathrm{P}=0.003)$. However, neither the MDA level nor the CAT level was significantly different between the two groups.

\section{The association between OS marker levels and oocyte quality}

Subsequently, we analyzed the association between GSH$\mathrm{Px}, \mathrm{SOD}$ or $\operatorname{Prdx} 4$ and oocyte quality in all participants 
Table 2 Oxidative stress marker concentrations in FF according to clinical pregnancy outcome

\begin{tabular}{lccc}
\hline Variable & Pregnant $(\mathrm{N}=42)$ & Non-pregnant $(\mathrm{N}=74)$ & $\mathrm{P}$ value \\
\hline $\mathrm{H}_{2} \mathrm{O}_{2}(\mu \mathrm{mol} / \mathrm{L})$ & $21.39 \pm 5.30$ & $23.61 \pm 2.89$ & $\mathrm{NS}$ \\
$\mathrm{GSH}(\mathrm{mg} / \mathrm{L})$ & $1.76 \pm 0.31$ & $2.13 \pm 0.19$ & $\mathrm{NS}$ \\
$\mathrm{GSH}-\mathrm{Px}(\mathrm{U} / \mathrm{mL})$ & $130.95 \pm 10.56$ & $100.83 \pm 5.40$ & $0.01^{\star}$ \\
$\mathrm{MDA}(\mathrm{mmol} / \mathrm{L})$ & $3.90 \pm 0.30$ & $3.87 \pm 0.16$ & $\mathrm{NS}$ \\
$\mathrm{CAT}(\mathrm{U} / \mathrm{mL})$ & $1.84 \pm 0.36$ & $1.37 \pm 0.19$ & $\mathrm{NS}$ \\
$\operatorname{SOD}(\mathrm{U} / \mathrm{mL})$ & $54.06 \pm 3.61$ & $43.87 \pm 1.76$ & $0.01^{*}$ \\
$\operatorname{Prdx4}(\mathrm{ng} / \mathrm{mL})$ & $22.26 \pm 1.65$ & $16.43 \pm 0.97$ & $<0.01^{\star *}$ \\
\hline
\end{tabular}

Values are mean $\pm \mathrm{SEM} ;{ }^{*}$, statistically significant $(\mathrm{P}<0.05) ;{ }^{* *}$, highly statistically significant $(\mathrm{P}<0.01)$, NS, not significant.

Table 3 The association between GSH-Px, SOD and Prdx4 levels and oocyte quality

\begin{tabular}{lccc}
\hline Variable & GSH-Px & SOD & Prdx4 \\
\hline Number of oocytes retrieved & $r=-0.209 ; P=0.118$ & $r=0.034 ; P=0.802$ & $r=0.015 ; P=0.911$ \\
Fertilization rate (\%) & $r=0.014 ; P=0.916$ & $r=0.307 ; P=0.020^{*}$ & $r=0.334 ; P=0.011^{*}$ \\
Cleavage rate (\%) & $r=0.193 ; P=0.150$ & $r=0.154 ; P=0.253$ & $r=0.141 ; P=0.294$ \\
Good quality embryo rate (\%) & $r=-0.042 ; P=0.754$ & $r=0.306 ; P=0.021^{*}$ & $r=0.326 ; P=0.013^{*}$ \\
\hline
\end{tabular}

Statistical analysis of the data was performed using the non-parametric Spearman test; ${ }^{*}$, statistically significant $(P<0.05)$.

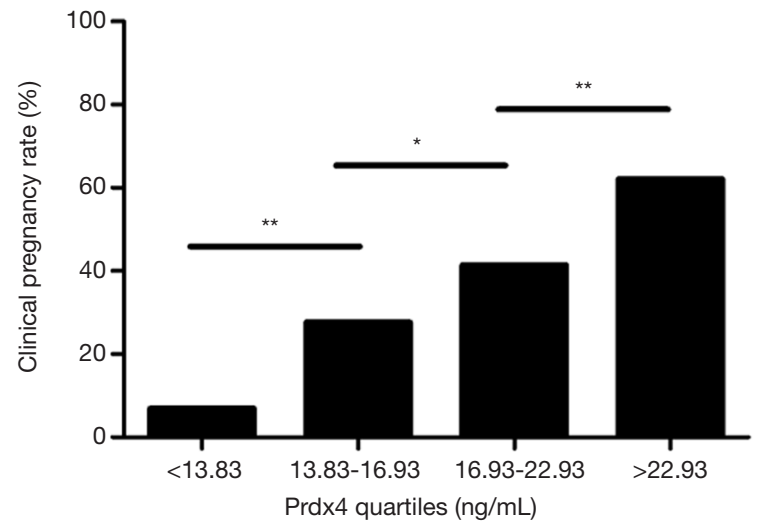

Figure 2 The clinical pregnancy rate was positively correlated with $\operatorname{Prdx} 4$ levels. All participants were divided into quartiles according to $\operatorname{Prdx} 4$ concentrations. The results showed that the clinical pregnancy rates were $6.9 \%, 27.59 \%, 41.38 \%$ and $62.07 \%$ in the four groups, respectively. *, statistically significant $(\mathrm{P}<0.05)$; **, highly statistically significant $(\mathrm{P}<0.01)$.
(Table 3). We found that the SOD levels were positively correlated with the fertilization rate $(\mathrm{r}=0.307 ; \mathrm{P}=0.020)$ and good quality embryo rate $(r=0.306 ; P=0.021)$, but not with the number of oocytes retrieved $(\mathrm{r}=0.034 ; \mathrm{P}=0.802)$ or the cleavage rate $(\mathrm{r}=0.154 ; \mathrm{P}=0.253)$.

The level of $\operatorname{Prdx} 4$, a newly identified OS marker, was positively correlated with both the oocyte fertilization rate $(\mathrm{r}=0.334 ; \mathrm{P}=0.011)$ and good quality embryo rate $(\mathrm{r}=0.326$; $\mathrm{P}=0.013)$ in all patients. However, no significant association was found between the $\operatorname{Prdx} 4$ level and the number of oocytes retrieved or the cleavage rate.

\section{Predictive ability of Prdx4 for clinical pregnancy}

To further investigate the association between the Prdx4 level and oocyte quality, we divided all participants into four groups by quartering the levels of $\operatorname{Prdx} 4(<13.38$, $13.83-16.93,16.93-22.93,>22.93 \mathrm{ng} / \mathrm{mL})$. Then, the clinical pregnancy rate was explored (Figure 2). The results 
Table 4 Oocyte quality and pregnancy outcomes according to $\operatorname{Prdx} 4$ quartiles

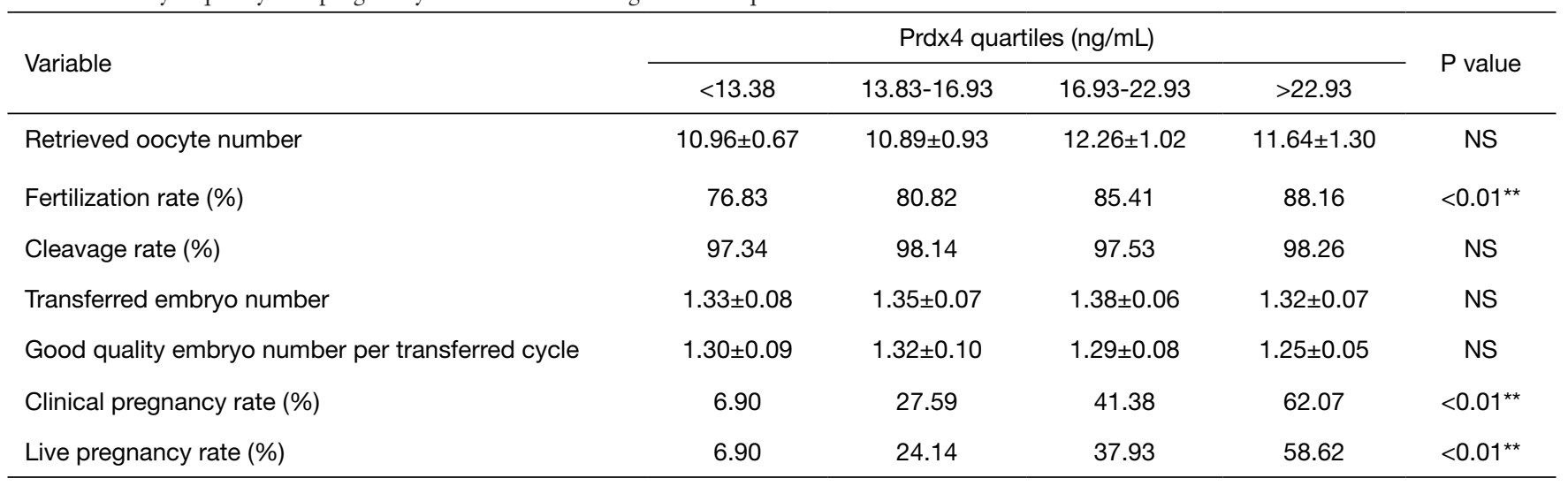

Values are mean $\pm S E M ;{ }^{* *}$, highly statistically significant $(P<0.01)$. NS, not significant.

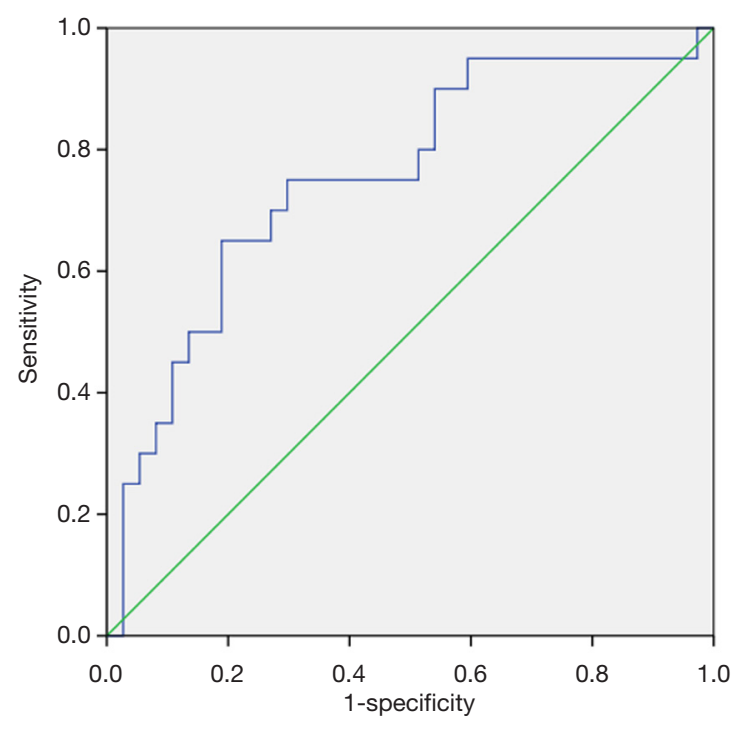

Figure 3 ROC curve for the $\operatorname{Prdx} 4$ levels in the prediction of clinical pregnancy.

showed that the pregnancy rate was positively correlated with the $\operatorname{Prdx} 4$ levels in a concentration-dependent manner in the four groups, with clinical pregnancy rates of $6.90 \%, 27.59 \%, 41.38 \%$ and $62.07 \%$ in the four groups, respectively.

Then, the oocyte quality in these four groups was analyzed, and the results are shown in Table 4. The fertilization rates, clinical pregnancy rates and live pregnancy rates were all significantly higher in the highest $\operatorname{Prd} \times 4$ quartile group than in the lowest quartile $(\mathrm{P}<0.01)$.

The predictive abilities of $\operatorname{Prdx} 4$ for clinical pregnancy were further analyzed with a ROC curve (Figure 3). $\operatorname{Prdx} 4$ showed a high accuracy for the prediction of clinical pregnancy with an AUC of 0.754 (95\% CI: 0.659-0.849). The Prdx 4 cutoff value for clinical pregnancy prediction was $22.30 \mathrm{ng} / \mathrm{mL}$ with a sensitivity of $65.0 \%$ and a specificity of $81.1 \%$.

\section{Discussion}

Since the identification of $\operatorname{Prdx} 4$ in human cells in 1997, several functions of $\operatorname{Prdx} 4$ have been reported, including its promotion of cell growth, involvement in cancer cell metastasis $(11,12)$, and prevention of virus-induced oxidative damage in respiratory epithelial cells. Many studies have identified $\operatorname{Prdx} 4$ as an antioxidant, suggesting that the $\operatorname{Prdx} 4$ expression level might be an effective marker of OS in the body (13) and proposed that $\operatorname{Prdx} 4$ can predict the incidence of some diseases (14), such as cardiovascular disease (15) and recurrent pregnancy loss. Recent studies have shown that $\operatorname{Prdx} 4$ is independently associated with the mortality of type 2 diabetes patients $(16,17)$. We have previously reported that $\operatorname{Prdx} 4$ is abundantly expressed in human ovarian GCs and can be associated with follicular development $(6,18)$. FF is mainly secreted by GCs, and in this study, we found that the $\operatorname{Prdx} 4$ protein is abundantly expressed in the FF of IVF patients.

Many studies have reported the effects of a variety of ROS and antioxidants in FF on IVF outcomes, although the results are controversial (19-23). In this study, GSH-Px, SOD and Prdx4 were all strongly correlated with the clinical pregnancy outcome (Table 2). Oocyte quality is widely accepted to be a major factor associated with IVF outcomes. Our results strengthen the assumption that oocyte quality 


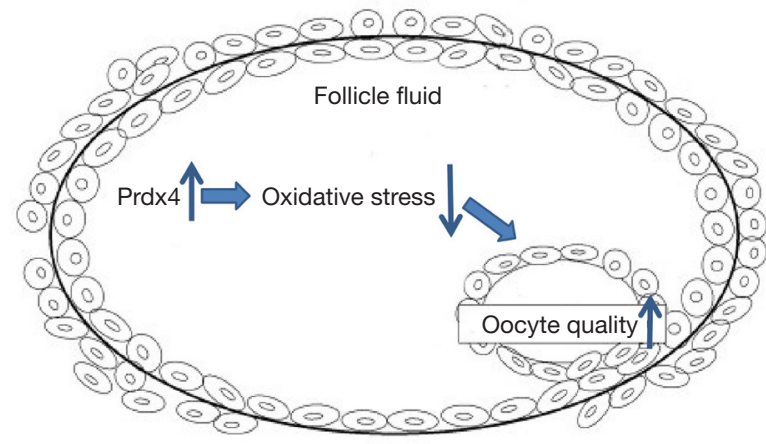

Figure 4 Schematic illustrating the possible roles of $\operatorname{Prdx} 4$ in follicular fluid. Upregulated expression of antioxidants, such as $\operatorname{Prdx} 4$, in the follicular fluid of IVF patients tends to improve oocyte quality by decreasing oxidative stress.

is associated with antioxidant levels in IVF patients. We analyzed the association between GSH-Px, SOD or Prdx4 and oocyte quality in the patients (Table 3). The results showed that SOD levels were positively correlated with fertilization ( $\mathrm{r}=0.307 ; \mathrm{P}=0.020)$ and good quality embryo $(\mathrm{r}=0.306 ; \mathrm{P}=0.021)$ rates. Our results differ from those of Liu et al. (24), who reported several years ago that there is no correlation between SOD and oocyte maturity, embryo quality, fertilization, or cleavage-stage embryo rates. The current conflicting findings may be attributed to the different methodologies used and endpoint outcomes examined. Furthermore, the studies were observational, and confounding variables, such as age and patient recruitment standard, may have influenced the results.

Recent studies have indicated that the Prdx family is associated with female reproduction $(25,26)$. The Prdx2 protein has been detected in human $\mathrm{FF}$ and found to be an important regulator in follicle atresia that functions by inhibiting GC apoptosis (25). The level of Prdx6 expression is increased in bovine oocytes and cumulus cells during the in vitro maturation (IVM) process. However, little is currently known about the function of $\operatorname{Prdx} 4$ in female reproduction. Accordingly, we first explored the relationship of $\operatorname{Prdx} 4$ expression with IVF outcomes. The results showed that $\operatorname{Prdx} 4$ levels were positively correlated with both the oocyte fertilization rate $(\mathrm{r}=0.334 ; \mathrm{P}=0.011)$ and the good quality embryo rate $(\mathrm{r}=0.326 ; \mathrm{P}=0.013)$. Furthermore, our study indicated that the pregnancy rate was positively correlated with $\operatorname{Prdx} 4$ levels in a concentration-dependent manner in the $\operatorname{Prdx} 4$ quartiles $(<13.38,13.83-16.93,16.93-22.93,>22.93 \mathrm{ng} / \mathrm{mL})$ (Figure 2). Increases in the fertilization rates, clinical pregnancy rates and live pregnancy rates with increasing Prdx4 was observed by quantiles (Table 4). Subsequently, the results indicated that $\operatorname{Prdx} 4$ had an area under the receiver operating characteristic curve (AUC) of 0.754 (95\% CI: 0.659-0.849) (Figure 3). While the cutoff level of $\operatorname{Prdx} 4$ should be interpreted with caution, the cutoff value for clinical pregnancy prediction was $22.30 \mathrm{ng} / \mathrm{mL}$ with a sensitivity of $65.0 \%$ and a specificity of $81.1 \%$. Based on the above results, we speculated that $\operatorname{Prdx} 4$, as an antioxidant, may be an effective biomarker for IVF patients to predict oocyte quality as well as subsequent pregnancy outcomes. However, the molecular mechanism is still unclear.

Previous studies have indicated that $\operatorname{Prdx} 4$ has two isoforms, a secreted type and an intracellular type. The former type can be secreted because the isoform lacks the $\mathrm{N}$-terminal peptide, while the intracellular type is mainly localized to the endoplasmic reticulum. We suspected that $\operatorname{Prdx} 4$ expressed in the FF of IVF patients is mostly the secreted type. Endogenous oxygen radicals are generated during the oocyte development process. In response to the production of these harmful oxygen radicals, secretion of thiol-dependent peroxidases, such as $\operatorname{Prdx} 4$, will increase to facilitate their removal. Intracellular removal of hydrogen peroxides by $\operatorname{Prdx} 4$ and secretion of the enzyme are hypothesized to be proportionally regulated in response to the surrounding oxidative stress (17). Thus, $\operatorname{Prdx} 4$, as a part of the antioxidant defense system, can be regarded as a marker of oocyte damage and may therefore be indirectly linked to oxidative stress or even IVF outcomes. It is speculated that higher expression of antioxidants, such as $\operatorname{Prdx} 4$, in the follicular fluid of IVF patients tends to indicate better oocyte quality by decreasing oxidative stress (Figure 4).

Previous studies have shown that during the oocyte IVM process, exogenous addition of antioxidants, such as $\beta$-cryptoxanthin, metformin, and CoQ10, can significantly reduce oxidative stress levels and improve oocyte quality and developmental potential $(27,28)$. Based on these study results, we hypothesized that exogenous addition of Prdx4 protein into oocyte IVM culture medium may promote oocyte quality. In addition, when a patient shows a high level of Prdx4 in FF and she has extra oocytes for donation, we may suppose a better ongoing pregnancy outcome of the oocytes and might tend to prioritize the use of her oocytes.

\section{Acknowledgments}

Funding: This work was supported by grants from the 
National Nature and Science Foundation of China (81701517), National Key Research and Development Program of China (2017YFC1001602, 2017YFC1001300), Jiangsu Province High Level Health Personnel Project (LGY2019066) and MerckSerono China Research Fund for Fertility Experts (CREATE-2016023).

\section{Footnote}

Reporting Checklist: The authors have completed the STROBE reporting checklist. Available at http://dx.doi. org/10.21037/atm-20-397

Data Sharing Statement: Available at http://dx.doi. org/10.21037/atm-20-397

Peer Review File: Available at http://dx.doi.org/10.21037/ atm-20-397

Conflicts of Interest: All authors have completed the ICMJE uniform disclosure form (available at http://dx.doi. org/10.21037/atm-20-397). The authors have no conflicts of interest to declare.

Ethical Statement: The authors are accountable for all aspects of the work in ensuring that questions related to the accuracy or integrity of any part of the work are appropriately investigated and resolved. The study was conducted in accordance with the Declaration of Helsinki (as revised in 2013). This study was approved by the Institutional Ethics Committee of The First Affiliated Hospital of Nanjing Medical University (No. 2016-SRFA038), and informed consent was obtained from all patients.

Open Access Statement: This is an Open Access article distributed in accordance with the Creative Commons Attribution-NonCommercial-NoDerivs 4.0 International License (CC BY-NC-ND 4.0), which permits the noncommercial replication and distribution of the article with the strict proviso that no changes or edits are made and the original work is properly cited (including links to both the formal publication through the relevant DOI and the license). See: https://creativecommons.org/licenses/by-nc-nd/4.0/.

\section{References}

1. Younis A, Clower C, Nelsen D, et al. The relationship between pregnancy and oxidative stress markers on patients undergoing ovarian stimulations. J Assist Reprod Genet 2012;29:1083-9.

2. Paszkowski T, Traub AI, Robinson SY, et al. Selenium dependent glutathione peroxidase activity in human follicular fluid. Clin Chim Acta 1995;236:173-80.

3. Algriany O, Bevers M, Schoevers E, et al. Follicle sizedependent effects of sow follicular fluid on in vitro cumulus expansion, nuclear maturation and blastocyst formation of sow cumulus oocytes complexes. Theriogenology 2004;62:1483-97.

4. Yiyenoglu OB, Ugur MG, Ozcan HC, et al. Assessment of oxidative stress markers in recurrent pregnancy loss: a prospective study. Arch Gynecol Obstet 2014;289:1337-40.

5. Abbasi A, Corpeleijn E, Postmus D, et al. Peroxiredoxin 4, a novel circulating biomarker for oxidative stress and the risk of incident cardiovascular disease and all-cause mortality. J Am Heart Assoc 2012;1:e02956.

6. Meng Y, Qian Y, Gao L, et al. Downregulated expression of peroxiredoxin 4 in granulosa cells from polycystic ovary syndrome. PLoS One 2013;8:e76460.

7. Iuchi $\mathrm{Y}$, Okada F, Tsunoda S, et al. Peroxiredoxin 4 knockout results in elevated spermatogenic cell death via oxidative stress. Biochem J 2009;419:149-58.

8. Mehmeti I, Lortz S, Elsner M, et al. Peroxiredoxin 4 improves insulin biosynthesis and glucose-induced insulin secretion in insulin-secreting INS-1E cells. J Biol Chem 2014;289:26904-13.

9. Yi N, Xiao MB, Ni WK, et al. High expression of peroxiredoxin 4 affects the survival time of colorectal cancer patients, but is not an independent unfavorable prognostic factor. Mol Clin Oncol 2014;2:767-72.

10. Nawata A, Noguchi H, Mazaki Y, et al. Overexpression of peroxiredoxin 4 affects intestinal function in a dietary mouse model of nonalcoholic fatty liver disease. PLoS one 2016;11:e0152549.

11. Fujii J, Ikeda Y, Kurahashi T, et al. Physiological and pathological views of peroxiredoxin 4. Free Radic Biol Med 2015;83:373-9.

12. Kim TH, Song J, Alcantara Llaguno SR, et al. Suppression of peroxiredoxin 4 in glioblastoma cells increases apoptosis and reduces tumor growth. PLoS One 2012;7:e42818.

13. Klichko VI, Orr WC, Radyuk SN. The role of peroxiredoxin 4 in inflammatory response and aging. Biochim Biophys Acta 2016;1862:265-73.

14. Rafiei S, Tiedemann K, Tabaries S, et al. Peroxiredoxin 4: a novel secreted mediator of cancer induced osteoclastogenesis. Cancer Lett 2015;361:262-70.

15. Hwang JA, Song JS, Yu DY, et al. Peroxiredoxin 4 as an 
independent prognostic marker for survival in patients with early-stage lung squamous cell carcinoma. Int J Clin Exp Pathol 2015;8:6627-35.

16. Ding Y, Yamada S, Wang KY, et al. Overexpression of peroxiredoxin 4 protects against high-dose streptozotocininduced diabetes by suppressing oxidative stress and cytokines in transgenic mice. Antioxid Redox Signal 2010;13:1477-90.

17. Gerrits EG, Alkhalaf A, Landman GW, et al. Serum peroxiredoxin 4: a marker of oxidative stress associated with mortality in type 2 diabetes (ZODIAC-28). PLoS One 2014;9:e89719.

18. Qian Y, Shao L, Yuan C, et al. Implication of differential peroxiredoxin 4 expression with age in ovaries of mouse and human for ovarian aging. Curr Mol Med 2016;16:243-51.

19. Luddi A, Capaldo A, Focarelli R, et al. Antioxidants reduce oxidative stress in follicular fluid of aged women undergoing IVF. Reprod Biol Endocrinol 2016;14:57.

20. Rahiminejad ME, Moaddab A, Ganji M, et al. Oxidative stress biomarkers in endometrial secretions: a comparison between successful and unsuccessful in vitro fertilization cycles. J Reprod Immunol 2016;116:70-5.

21. Combelles CM, Holick EA, Paolella LJ, et al. Profiling of superoxide dismutase isoenzymes in compartments of the developing bovine antral follicles. Reproduction 2010;139:871-81.

Cite this article as: Yi Q, Meng C, Cai LB, Cui YG, Liu JY, Meng Y. Peroxiredoxin 4, a new oxidative stress marker in follicular fluid, may predict in vitro fertilization and embryo transfer outcomes. Ann Transl Med 2020;8(17):1049. doi: 10.21037/atm20-397
22. Pasqualotto FF, Sobreiro BP, Hallak J, et al. Sperm concentration and normal sperm morphology decrease and follicle-stimulating hormone level increases with age. BJU Int 2005;96:1087-91.

23. Yalcinkaya E, Cakiroglu Y, Doger E, et al. Effect of follicular fluid NO, MDA and GSH levels on in vitro fertilization outcomes. J Turk Ger Gynecol Assoc 2013;14:136-41.

24. Liu F, He L, Liu Y, et al. The expression and role of oxidative stress markers in the serum and follicular fluid of patients with endometriosis. Clin Exp Obstet Gynecol 2013;40:372-6.

25. Yang S, Luo A, Hao X, et al. Peroxiredoxin 2 inhibits granulosa cell apoptosis during follicle atresia through the NFKB pathway in mice. Biol Reprod 2011;84:1182-9.

26. Park SJ, Kim JH, Lee DG, et al. Peroxiredoxin 2 deficiency accelerates age-related ovarian failure through the reactive oxygen species-mediated JNK pathway in mice. Free Radic Biol Med 2018;123:96-106.

27. Park YG, Lee SE, Son YJ, et al. Antioxidant $\beta$-cryptoxanthin enhances porcine oocyte maturation and subsequent embryo development in vitro. Reprod Fertil Dev 2018;30:1204-13.

28. Rodrigues-Cunha MC, Mesquita LG, Bressan F, et al. Effects of melatonin during IVM in defined medium on oocyte meiosis, oxidative stress, and subsequent embryo development. Theriogenology 2016;86:1685-94. 